\title{
Protée
}

\section{À corps ouverts. Changement et échange d'identités dans la Capoeira et le contact improvisation}

\section{Ann Cooper Albright}

Volume 29, numéro 2, 2001

Danse et altérité

URI : https://id.erudit.org/iderudit/030624ar

DOI : https://doi.org/10.7202/030624ar

Aller au sommaire du numéro

Éditeur(s)

Département des arts et lettres - Université du Québec à Chicoutimi

ISSN

0300-3523 (imprimé)

1708-2307 (numérique)

Découvrir la revue

Citer cet article

Albright, A. C. (2001). À corps ouverts. Changement et échange d'identités dans la Capoeira et le contact improvisation. Protée, 29(2), 39-49.

https://doi.org/10.7202/030624ar
Résumé de l'article

Cet article tente de montrer comment deux formes particulières de la danse contemporaine, la Capoeira et le contact improvisation peuvent fournir des modèles - et même des technologies physiques - à partir desquels théoriser une altérité incarnée. Il montre comment ces formes de danse proviennent de cultures et d'histoires spécifiques mais aussi comment, dans leurs manifestations contemporaines, elles possèdent leur propre logique qui nous force à repenser notre compréhension des corps et des cultures. 


\title{
À CO RPS O U VERTS: CHANGEMENT ET ÉCHANGE D'IDENTITÉS DANS LA CAPOEIRA ET LE CONTACT IMPRO VISATION \\ Traduit de l'anglais par Jean-Pierre Vidal \\ ANN COOPER ALBRIGHT
}

\begin{abstract}
Un danseur solitaire entre dans le cercle, d'abord en rampant, puis en roulant et finalement en valsant jusqu'au centre. Il trébuche presque, se retourne et s'arrête, regardant de tous côtés et plongeant son regard dans celui de quelqu'un, n'importe qui. Un autre danseur accepte cette invitation silencieuse et entre dans la danse, bondissant dans le cercle comme un météore qui traverse une galaxie en tournoyant. Il lance son corps dans une furieuse série de mouvements. Une quelconque forme de gravité (ou peutêtre simplement l'épuisement) l'attire dans l'orbite de l'autre danseur et comme ils se retournent tous deux, leurs mains se rencontrent dans une recherche d'équilibre. Gonflés à bloc par cette conjonction de leurs forces, ils dessinent un chemin qui obéit à sa propre logique dans l'espace. Ils sont poussés par l'échange qu'ils font de leur élan, de leur poids et de leur souffle, se mouvant si rapidement à travers formes et espace qu'ils doivent abandonner tout désir de prendre des décisions et se contentent simplement d'observer leur périple à mesure qu'il se déploie. Leurs échanges finissent par devenir plus lents et plus retenus, atteignant finalement un fragile équilibre, une tête reposant dans le cou de l'autre.

Ils s'accroupissent, en attente, encerclés par les rythmes syncopés et les voix des gens qui les entourent. Leurs mains se rencontrent à peine, brièvement, mais leurs yeux sont rivés, maintenant le contact, même quand ils culbutent en se recevant sur les mains. À nouveau sur leurs pieds, ils se balancent d'avant en arrière à l'unisson, gardant leurs articulations déliées et leur imagination souple. La spécularité fluide qui les unit change de dynamique quand l'un d'eux brusquement bouge et que l'autre relève le défi. Cela déclenche une réaction joueuse qui leur fait explorer les possibilités d'un échange de mouvements qui flirte avec le contact physique, mais sans jamais y tomber.
\end{abstract}

Ces deux descriptions de mouvements en duo - cette rencontre d'un «autre» en mouvement - cartographient le genre de rencontres physiques dont je traiterai dans cet article. Le premier passage décrit un moment de contact improvisation. Le second un moment de Capoeira. En surface, ces formes représentent des histoires culturelles disparates et des dynamiques d'interaction physique fort différentes. Après tout, l'une concerne une immersion dans une union physique, l'autre parle de la façon dont on émerge (en toute sécurité) d'un combat physique, mais les deux formes ont pris possession des imaginations cinétiques des danseurs contemporains et ont influencé les vocabulaires du mouvement à l'échelle 
mondiale. Les deux formes représentent aussi, et de plus en plus, des phénomènes globaux. Le contact improvisation et la Capoeira ont surgi de moments de confusion et d'effervescence historique et culturelle et les deux formes conservent encore dans leurs stratégies physiques des éléments issus de ces moments de résistance et de rébellion. Et pourtant, les deux formes ont aussi continué de se réinventer, en incorporant, au sens fort, des préoccupations contemporaines dans leurs vocabulaires de mouvements. Et le plus important, c'est que les deux formes ont contribué à la remise en question des notions conventionnelles d'identité et de géographie, créant à la place une expérience somatique qui reconstruit de l'intérieur les notions que nous avons de l'altérité. Comme je vais tenter de le montrer dans cet article, la Capoeira et le contact improvisation ont tous deux donné forme à une pratique physique et métaphysique qui peut nous aider à survivre et même à grandir dans notre monde interculturel sans cesse changeant et plein de défis.

Cet article tentera de formuler la forte conviction, viscéralement ancrée en moi, que quelque chose de vraiment important est en train de se passer ici. La conviction que, au milieu de ces échanges improvisés d'attention, de gestes, de sueur et d'énergie, on s'exerce à confronter un "autre», de telle façon que les distinctions habituelles entre le moi et l'autre se trouvent confondues. C'est ici la première fois que je tente de traduire en termes théoriques mon implication physique dans ces formes de mouvements. Le ton évangélique de cet écrit vient de la conviction que l'expérience de la danse et de la performance de contact improvisation pendant la majeure partie de ma vie adulte et ma formation en Capoeira ces dernières années m'ont donné une perspective intéressante sur la façon de cerner, le plus spécifiquement possible, la subjectivité et l'altérité. Comme les discussions, apparemment sans fin à propos de la dimension politique de l'identité, qui agitaient aussi bien les cercles universitaires que les communautés artistiques, me faisaient passer par des alternances de confusion, d'allégresse, de dépression ou carrément d'hébétude, je me suis surprise à trouver refuge dans les interactions corps à corps des studios où je pratique. Je ne veux pas dire par là que ces relations physiques sont nécessairement plus faciles, moins complexes ou sans problème. Mais il me semble que, même si les questions de diversité humaine ne se bornent jamais au corps dans ce qu'il a de purement physique - elles sont en effet toujours prises dans des contextes sociaux et des structures de représentation -, c'est par nos corps qu'elles se manifestent. En tant que féministe, je suis consciente que les corps sont profondément façonnés par des attitudes culturelles et des conditions économiques. En tant que danseuse, je suis consciente que les corps peuvent aussi être réentraînés et consciemment re-théorisés. Je suis persuadée que le comportement non verbal joue un rôle significatif dans la négociation de l'identité sociale. La façon dont nous nous abordons les uns les autres, dont nous marchons, parlons et dansons ensemble a énormément de signification.

Comme nous le savons tous, la différence est affaire de pouvoir. Ce qui veut dire que la différence culturelle est inscrite (le plus souvent) sur le corps selon des modalités qui sont ensuite lues en termes d'identités sociales. La race, le sexe, la classe, l'ethnie, les préférences sexuelles, l'âge et les capacités sont (encore), tous autant qu'ils sont, des marqueurs essentiels de la différence en ce début de $21^{\mathrm{e}}$ siècle. Mais toutes ces différences représentent des manifestations d'une structure binaire de la différence caractéristique de l'épistémologie occidentale. L'esprit est séparé du corps et les deux sont ensuite figurés en termes d'identité (c'est la célèbre formule de Descartes "Je pense, donc je suis») et d'altérité, celle-ci se trouvant intériorisée comme une incarnation. Dans les deux dernières décennies, les féministes nous ont fourni une véritable litanie d'exemples montrant clairement à quel point la séparation du corps et de l'esprit peut être prévalante dans notre culture. Comme elles l'ont démontré hors de tout doute, les idéologies religieuses et les philosophies fondamentales de la civilisation occidentale ont d'abord construit puis cherché à naturaliser un 
paradigme dualiste divisant le monde en catégories oppositionnelles, telles que corps/esprit, nature/ culture, privé/public, spiritualité/corporéité et expérience/savoir. Ces polarisations schématiques ont à leur tour créé des hiérarchies inévitables qui ont positionné le corps comme l'«autre» matériel de la transcendance propre à l'esprit.

Dans son livre Subjectivity, Identity and the Body, Sidonie Smith décrit l'héritage misogyne de cette désincarnation du sujet universel et montre comment il s'est développé en une séparation en genre du sujet et de l'autre, l' "Autre" se réduisant à n'être rien d'autre que son corps de femme, avec en guise d'identité un vide matériel ${ }^{1}$. Judith Butler affirme sensiblement la même chose quand elle prétend que:

By defining women as "Other», men are able through the shortcut of definition to dispose of their bodies, to make themselves other than their bodies [...] From this belief that the body is Other, it is not a far leap to the conclusion that others are their bodies, while the masculine "I» is the noncorporeal soul. The body rendered as Other - the body repressed or denied and, then, projected - reemerges for this «I» as the view of others as essentially body. Hence, women become the Other; they come to embody corporeality itself. 2

Cela n'arrive pas seulement aux femmes, bien sûr; les gens de couleur, les gays, les exclus de toutes sortes aussi bien que les personnes handicapées ont historiquement été rivés aux conditions matérielles de leurs corps, lesquels structuraient ainsi une identité qui se construisait perpétuellement comme oppressivement et bassement physique, comme un manque d'identité - un manque de contenance morale, spirituelle et sociale. Incontestablement, ce sont les individus que la société associe à l'être corporel qui subissent le plus fortement les conséquences répressives de cette tradition.

Les analyses féministes de la séparation constante de l'esprit et du corps dans les cultures occidentales ont jeté les bases critiques des discussions contemporaines portant sur l'oppression sociale fondée sur les catégories binaires de moi et d'autre, de normal et de différent. Ces catégories de différence inscrites dans le corps - différences de race, de classe, d'origine ethnique aussi bien que différences de capacités physiques et de sexualité - ont radicalement influencé l'imagination populaire et le discours académique. Et pourtant, après plusieurs décennies passées à dresser brillamment et dans tous ses détails le catalogue des diverses formes d'oppression sociale, nous devons nous demander: "Quelle différence la différence a-t-elle bien pu faire?». Je ne voudrais pas par là me montrer facétieuse, bien au contraire. Je suis profondément convaincue des possibilités de libération, individuelles et collectives, de ces analyses culturelles. Mais ce que je montre ainsi du doigt, c'est la prochaine étape. Car il devra nécessairement y avoir une autre étape. Le problème avec un relevé encyclopédique des différences c'est qu'elles font courir le risque de réifier l'opposition binaire, réduisant ainsi la différence au fait, statique et monolithique, qu'elle est la différence. Plutôt que de considérer la différence comme une position arrêtée, je plaide pour un positionnement (et, par extension, un re-positionnement) qui soit toujours en mouvement. Je présenterai, tout au long de cet article, la Capoeira et le contact improvisation comme des exemples de duos - le "pas de deux", cette confrontation physique avec l'autre - dans lesquels la différence peut être reconnue mais aussi traversée, déplacée, côtoyée, approchée, soulignée, dépassée, respectée, etc. Ces formes représentent des échanges physiques qui se situent au-delà de la différence, des échanges au cours desquels le fait corporel de la différence se trouve célébré et devient mobile.

Avant de m'attacher aux caractéristiques de ces formes de mouvement et comme j'ai peut-être rendu certains lecteurs nerveux, je voudrais mettre les choses au point. Je ne défends pas une quelconque utopie ni ne préconise un retour naif à un humanisme d'avant le poststructuralisme. Les deux dernières décennies ont opéré une rupture radicale dans les visions du monde trop complaisantes et je crois que l'hésitation que nous éprouvons toutes et tous, ce moment de lucidité qui nous fait nous arrêter pour réfléchir quand nous parlons ou écrivons est capital pour toute 
restructuration de la dynamique des privilèges et du pouvoir. Je suis profondément convaincue que toutes ces luttes de libération doivent se poursuivre, qu'en fait elles sont même plus importantes que jamais. Mais je suis également sensible au fait qu'une conscience trop aiguë de la différence amène les gens à développer une conscience de soi qui peut s'avérer absolument débilitante, avec pour résultat que chacun se retire dans son coin, craignant tant de choquer ou d'être choqué que le gouffre entre nous tous ne fait que s'agrandir. Comment s'entraîner à franchir cette distance qui nous sépare? Comment encourager le désir de jouer avec la différence? Comment passer des idées à la pratique? Je crois que nous devons courir le risque de l'échec, risquer la gêne et la maladresse, risquer d'être mal à l'aise et de nous faire marcher sur les pieds, pour pouvoir nous propulser au-delà de la coupure métaphysique qui sépare le moi de l'autre. Les premiers gestes qui inaugurent un nouveau partenariat sont rarement aisés, mais nous devons malgré tout courir le risque et demander à l'autre de nous accorder cette danse.

Le contact improvisation est une forme de danse qui a été développée au début des années soixante-dix par un groupe de personnes intéressées à expérimenter la danse produite par l'échange de poids entre deux individus ou plus. Dans le contact improvisation, le mouvement est structuré par les lois physiques spécifiques qui régissent les échanges entre corps: les dynamiques changeantes du poids, de l'espace, de l'élan et de la force. Bien entendu, la façon dont nous expérimentons cette combinaison de divers éléments physiques est idéologique et déterminée par l'histoire, et les ramifications culturelles de cette forme de danse peuvent changer d'un instant à l'autre, d'une danse à l'autre et d'une année à l'autre. À ses débuts, le contact improvisation incarnait les nombreuses questions concernant le moi, l'indépendance, la communauté et le changement, emblématiques des années soixante ${ }^{3}$. Commençant dans un état de partage de responsabilités quant au poids de chacun (un état que les praticiens du contact improvisation décrivent comme le fait d'être «mutuellement dépendant» quant à son poids, c'est-à-dire de s'appuyer l'un sur l'autre de telle façon qu'aucun des deux individus ne porte entièrement son propre poids ou celui de son partenaire), les danseurs se guident sur ce point de contact qui progressivement tourne autour de leurs corps et se déplace dans l'espace. Influencé par les arts martiaux asiatiques et les release techniques contemporaines, l'apprentissage du mouvement propre à cette forme de danse vise à donner aux danseurs les habiletés qui leur permettront de suivre le déplacement de ce point de contact à travers leur poitrine, en descendant vers le ventre, le long des cuisses, puis en remontant dans le dos jusqu'au sommet de la tête. Les techniques physiques qui permettent de rouler, d'apprendre à quel moment donner ou retenir son poids, et comment accepter le poids de l'autre, aussi bien que de savoir tomber sans danger, permettent aussi l'improvisation - ce choix fait en commun d'une exploration de l'instant imprévisible -, cette improvisation qui constitue le fondement créatif de l'engagement du danseur dans le duo.

L'histoire de la Capoeira est plus longue et suscite plus de controverses que celle du contact improvisation et l'histoire que l'on vous raconte dépend en grande partie de celui ou de celle qui la raconte ou l'écrit. Mais la plupart des gens sont d'accord pour dire que la Capoeira est une forme de danse martiale afrobrésilienne qui a pris naissance à l'époque coloniale, quand les propriétaires terriens européens eurent à peu près complètement épuisé la réserve de maind'œuvre indigène et commencèrent à importer des esclaves d'Afrique, en particulier des régions du Congo et de l'Angola. Sous l'œil des maîtres européens, l'entraînement traditionnel des guerriers évolua vers une forme de combat à deux basé sur une succession rythmée d'attaques et de parades. Ce pas de deux martial se déroulait à l'intérieur d'un cercle protecteur (la roda) dont les rythmes changeaient pour signaler les dangers que pouvait représenter la surveillance des maittres. Rapidement, la dynamique du combat se transformait en mouvements moins menaçants (une simple danse). Plus tard, avec 
l'abolition de l'esclavage et l'urbanisation accélérée, la Capoeira a évolué encore une fois, mais dans le sens d'une sorte de combat de rue; elle inaugurait ainsi sa longue association avec la culture urbaine des jeunes. De fait, ce lien est encore évident aujourd'hui dans les divers combinés de Capoeira et de hip-hop que l'on peut voir sur les places et aux coins des rues, partout en Amérique du Sud et du Nord, en Europe et en Asie. Au milieu du 20e siècle, au Brésil, la Capoeira a fini par se structurer et se codifier de plus en plus avec l'ouverture d'écoles et d'académies vouées à former les jeunes au «jeu» de la Capoeira. À la fois combat et danse, la Capoeira a fait figure, tout au long de son histoire, de résistance improvisée, non seulement à l'endroit de l'institution coloniale de l'esclavage mais aussi dans le cadre de la dynamique plus locale du pouvoir économique et du rapport de force entre les races. Cette «résistance» n'est pas seulement une question de place prise par la Capoeira dans l'histoire du Brésil, mais concerne aussi la façon dont une certaine subversion peut effectivement s'inscrire dans l'expérience physique de cette forme.

Les divers rapports qu'entretient actuellement le contact improvisation avec un certain nombre de pratiques «alternatives» (le tai-chi, le Body-Mind Centering, le mouvement authentique, l'aïkido, etc.) et la façon dont la Capoeira est liée à la vie de la rue et à la culture des jeunes situent ces formes de danse dans les marges des espaces institutionnels, qu'ils soient gouvernementaux, universitaires ou artistiques. À part quelques exceptions, le contact improvisation est généralement enseigné par une population fluctuante de professeurs et d'interprètes migrants. Comme des abeilles ouvrières allant de ville en ville pour polliniser le milieu de la danse partout dans le monde, ces danseurs offrent leurs compétences à des danseurs formés dans les divers styles de danse en vogue à un certain moment dans leur propre culture. Ces ateliers vont généralement du week-end intensif au stage d'une ou deux semaines. À la différence de nombreuses autres formes de danse, le contact improvisation est rarement enseigné sur une base régulière. La Capoeira présente cette même caractéristique d'être enseignée elle aussi par des maîtres itinérants. Bien que dans certains pays la Capoeira ait été institutionnalisée (c'est, par exemple, le cas au Brésil et à New York), le plus gros de la transmission corps à corps de cette forme de danse s'effectue lors d'échanges plus informels et par des réseaux locaux. La jam contact, comme la roda de Capoeira, est un espace d'expérimentation ouvert aussi bien aux débutants qu'aux pratiquants confirmés.

Même si l'entrainement que ces formes de danse nécessitent peut paraître (en surface, du moins) plus informel que, par exemple, le ballet ou le karaté, et même si elles ont gardé des traits qui leur viennent de leurs histoires égalitaires et libertaires, le contact improvisation comme la Capoeira ont su raffiner des habiletés précises qui constituent une expérience somatique fondatrice capable de survivre aux priorités individuelles et aux différences régionales. De façon ironique, même si le contact improvisation et la Capoeira visent par leur entraînement des fins différentes, la discipline physique de base est remarquablement semblable sur plusieurs points. Les deux travaillent (en particulier dans leurs phases préliminaires) à développer l'endurance physique, en mettant surtout l'accent sur la force du haut du corps et la capacité de faire porter tout le poids sur des parties du corps inusitées (mains, tête, coudes, etc.). Il est très intéressant de remarquer que même si cette force donne au danseur un sentiment rafraîchissant d'ardeur sauvage et d'énergie, on ne lui permet jamais de se développer au point de n'être plus qu'une façon contractée, raide ou compacte de se servir de ses muscles. Dans le contact improvisation, on se sert de cette force pour se donner suffisamment d'élan pour soulever son partenaire ou le soutenir de façon à garder le point de contact en mouvement dans l'espace. Dans le contact improvisation, le rapport avec le partenaire opère toujours en boucle et il est rare d'y voir cette figure classique de la danse dans laquelle l'un des partenaires se contente de soulever l'autre. Dans la Capoeira, on utilise la force pour prolonger le mouvement du partenaire et le relancer. C'est pourquoi les blocages durs et directs (comme on en 
voit dans certains arts martiaux) restent rares. Habituellement on tente d'éviter l'attaque (en se penchant ou en reculant devant le pied ou la tête) pour pouvoir se retourner et reprendre le combat à partir d'une position plus avantageuse. Ce genre de force reste stratégique et s'attache essentiellement à modeler les structures de résistance et de fluidité que les mouvements des deux corps font surgir entre eux. Dans les deux formes de danse, on utilise la force pour produire un synchronisme esthétique des énergies dans lequel le mouvement de l'un suscite une réaction fluide chez l'«autre».

Cette impression de fluidité au cœur de la force est une des contradictions provocantes qui lient la Capoeira et le contact improvisation. Dans la convaincante analyse qu' elle propose de la Capoeira dans «Headspin: Capoeira's Ironic Inversions», Barbara Browning présente ces contradictions comme une sorte d'ironie: "the no in the yes, the big in the little, the earth in the sky, the fight in the dance " 4 . Et on pourrait ajouter: la fluidité au cœur de la force. Rendus conscients de cette fluidité par leur formation, les pratiquants du contact improvisation et de la Capoeira en retirent, je crois, une certaine intuition quant aux changements et échanges d'identités que l'on peut expérimenter sans sacrifier son rapport personnel à l'espace. Dans le fascinant article où il relie la pensée de Lévinas à la pratique du contact improvisation, sous le titre particulièrement judicieux de «(In) the In-Between» [au cœur de l'entredeux], David Williams décrit cette rencontre comme un «in-between or go-between, [it] is another space in which the "I" is both implicated and (re)conceived; it is the articulation of meeting-in-difference " 5 .

Williams continue de recenser les possibilités existentielles de cette forme de danse en prétendant que:

For each of the partners, contact constitutes the possible coexistence of form and spontaneity, rules-ofthe-game and dance, cause and effect, center and margin, proximity and distance. It is the "play" within the obdurate fixity of corporeal identities, its "give», its supple-ment, its différance: the unstable borderlands where an ethics of alterity occurs. ${ }^{6}$
Derrière cette importante dimension existentielle, il y a une pratique physique basée sur la désorientation, la curiosité et la volonté d'affronter l'«autre» à la fois intérieurement et extérieurement. Les formules de Williams décrivent les effets d'une expérience somatique dans laquelle les limites habituelles entre les corps et le sentiment d'une identité fixe se trouvent suspendues. Par exemple, l'un des tout premiers éléments que l'on montre aux étudiants c'est le point de contact. En face de son partenaire, l'étudiant vient presser le bout de ses doigts contre ceux de l'autre. Attentif à la façon dont l'énergie de tout le corps de son partenaire peut circuler de sa colonne vertébrale à ses bras puis à ses doigts, il attend. Il attend et observe ce point de contact qui va finir par bouger. Ce point fait de la rencontre de deux énergies, on le désigne parfois comme un «troisième esprit» et c'est sur lui que leur attention mutuelle dès lors se concentre. Le comportement des deux partenaires va désormais se mouler sur son périple spatial et rythmique. Au début, il peut sembler facile de distinguer celui qui mène de celui qui suit mais, avec le temps et la pratique, le remplacement de l'un par l'autre se change en un échange si rapide et si subtil que ces catégories de meneur et de mené commencent à perdre tout sens. Les oppositions binaires se trouvent ainsi subverties à mesure que l'attention se concentre sur le jeu spatial et tactile qui se déroule entre les deux danseurs. David Williams décrit cette idée abstraite en des mots qui se traduisent aisément en termes de dynamique du partenariat qui se développe dans le contact improvisation.

The crucial factor here is not how many ways two different units can relate to each other, but recognition that this «third element» is not a unit but an axis, not an entity but a state of being, less a relationship than an act of relating. 7

Les plaisirs très réels d'une danse contact sophistiquée, on les trouve dans les moments de suspension ou de chute en commun, dans cette magie apparente avec laquelle on exécute spontanément des mouvements compliqués. Cette connexion réciproque 
est le «chi» du contact improvisation, le lieu d'une incroyable fluidité et d'une fusion intuitive des énergies. Bien sûr, l'un des partenaires peut rompre le contact à n'importe quel moment au cours de la danse, soit pour se séparer de son partenaire (de façon temporaire ou permanente), soit pour revendiquer en quelque sorte sa qualité d' "auteur» de tel ou tel mouvement par lequel il conduit son partenaire dans une direction bien spécifique. Une partie de l'improvisation consiste à apprendre à affronter ouvertement ces situations, avec curiosité et non dans un esprit de confrontation. L'improvisation vous apprend à réagir sans jamais devenir réactionnaire.

Au début, les risques sont minimes. Doigts contre doigts, tête contre tête, dos à dos, les exercices préliminaires de contact amènent une prise de conscience de la façon dont des corps différents réagissent aux mouvements les plus simples qu'on leur fait faire. Lorsqu'ils deviennent plus habiles physiquement, les étudiants sont finalement initiés à des exercices qui mettent l'accent sur l'art de tomber sans être tendu et de confier tout leur poids à une autre personne, ainsi que sur la dés/orientation visuelle et spatiale. Cette dernière discipline joue un rôle capital dans la façon dont se produit l'échange d'identités dont Williams parle dans son texte. Même des exercices en apparence aussi simples que celui qui consiste à se laisser rouler au sol avec une autre personne peuvent s'avérer extrêmement désorientants. Ces divers exercices exigent que l'on se serve de ses yeux d'une façon très différente. Dans le contact improvisation, en effet, on apprend à voir non pas en fixant son regard sur les gens ou sur les objets environnants mais plutôt en gardant les yeux immobiles dans leurs orbites et en s'en remettant à une vision périphérique plus détendue. Cette légère modification du champ de vision change radicalement l'expérience que l'on a de l'espace. Rouler, tournoyer et se retrouver la tête en bas dans une pièce où d'autres personnes se déplacent en même temps de toutes sortes de façons me force à négocier physiquement un champ chaotique d'allées et venues dans lequel je dois renoncer à toute impression stable d'avoir une "position" précise dans l'espace. Il ne reste plus, dès lors, que l'impression de ne se situer que visà-vis des autres qui eux-mêmes, bien entendu, sont aussi en mouvement dans l'espace. Cette façon de danser dans tous les sens et les uns au milieu des autres peut donner beaucoup de plaisir et créer des situations plutôt cocasses, mais elle suggère aussi une autre façon d'être au monde avec les autres - une sorte de danse existentielle dans laquelle ce perpétuel changement de position permet d'envisager une certaine réorganisation des relations sociales.

Le contact improvisation implique un désir de danser dans un état de désorientation. À la différence de bien des types de danse, le contact improvisation est affaire de déséquilibre; il se fonde sur le plaisir d'une certaine perte de contrôle sur ses propres mouvements, à la fois physiquement et psychologiquement. Je pense que cette acceptation de vivre le vertige produit par un échange constant des poids corporels, les chutes inévitables et les moments d'intimité quand on rattrape l'autre ou qu'il nous rattrape, peut affecter en profondeur nos présupposés purement intellectuels quant à la stabilité des catégories de «moi» et d'«autre». Même si beaucoup de mes exemples pourront sembler à des universitaires n'être que des talents de danseur passablement excentriques (après tout, la plupart des universitaires ne sont guère intéressés à se laisser rouler sur le sol), les effets de ce travail somatique sont frappants et profonds. Dans un environnement universitaire où l'on parle beaucoup de respect mutuel, d'acceptation des différences et de construction de l'espace commun et où l'on exerce une grande activité intellectuelle à ce sujet, les étudiants qui suivent les cours de danse contact font tout cela, eux, et sur le plancher des vaches.

Le contact improvisation incite non seulement à prendre des engagements vis-à-vis d'un autre corps, mais amène aussi une meilleure compréhension de l'«autre» en soi. S'il faut en croire Elizabeth Grosz quand elle déclare que: «alterity is the very possibility and process of embodiment ${ }^{8}$, le travail somatique individuel qui fait partie intégrante de la formation en 
contact improvisation montre bien à quel point cette altérité intérieure est liée à l'altérité extérieure du partenaire. Par exemple, je fais souvent appel, au début de mon cours de contact improvisation, à un exercice appelé «the stand». Il s'agit d'un moment où les étudiants, après divers déplacements dans l'espace, en arrivent à rester immobiles, debout. Cela leur donne la possibilité d'éprouver les sensations de la sueur et de l'air sur leurs bras, de leur cœur qui bat, du rythme de leur pouls dans diverses parties de leur corps - une façon d'éprouver les subtils changements de poids et d'activité musculaire, une occasion de vivre son corps comme à la fois lié au sol par les pieds et déployé dans l'espace jusqu'à la tête - c'est un moment de réflexion sur la position de l'être humain, suspendu entre ciel et terre.

Plus tard dans l'exercice, je leur demande d'imaginer qu'ils ouvrent les pores de leur peau de façon à laisser entrer le monde dans l'espace de leur corps. Cette image de son propre corps comme faisant partie du paysage envisagé comme un tout, plutôt que comme le véhicule qui se déplace à travers ce paysage et le dispose à son gré, donne des résultats physiques évidents dans le relâchement du tonus musculaire du corps. Mais il se produit aussi là une profonde réorganisation psychique. En effet, si le monde est déjà à l'intérieur du corps, alors la distinction entre moi et l'autre est bien moins nette. La peau n'est plus tant cette barrière infranchissable qui défend l'intégrité du corps (comme l'affirmaient toutes les métaphores de désinfection qui dans les années cinquante dominaient aussi bien la sphère domestique [attention à ces germes qui infestent votre cuisine et votre salle de bain] que le climat politique international [prenez garde à la diffusion du virus du communisme]) que l'organe sensoriel qui rapproche le monde. En agissant sur notre imaginaire somatique, nous pouvons remettre en cause les notions d'identité que notre culture nous a léguées. Plutôt que d'obéir au paradigme colonialiste de l'individu, poussé par sa volonté et sa détermination à s'inscrire dans le monde et à en revendiquer une part (se tenir debout sur ses deux pieds, faire sa marque, etc.), le moi devient une simple partie interdépendante de ce monde et se répand à travers lui, s'écoule avec lui.

Ouvrir son corps au monde est un geste profondément généreux. C'est le «don» que Williams décrit comme le jeu dans l'inflexible fixité des identités physiques. Car à mesure que les corps commencent à devenir plus mobiles, explorant l'espace et échangeant des mouvements entre eux, cette fluidité des frontières devient la danse. Cette générosité supprime les divisions traditionnelles du pouvoir en refusant de croire à l'imperméabilité du moi prisonnier de son corps. Le danseur dit: «Viens, prends mon corps, entre en moi, sers-toi de moi, laissetoi rouler sur moi, laisse-moi te soutenir, m'appuyer sur toi». De façon très physique, les danseurs contact offrent leurs corps à leurs partenaires. Mais il s'agit d'une invite, pas d'une humiliation. Car même si les frontières sont fluides, ce n'est pas une chute libre existentielle. Au cour de tous ces échanges, l'individu reste encore branché sur ses propres sensations physiques et sur la rassurante sensation de la gravité au moment même où il se fait soulever; c'est une solide prise de terre qui le garde en sécurité alors même qu'il se lance à travers l'espace et le temps.

Ce «don», cet abandon de toute identité stable, est aussi en «jeu» dans la Capoeira. Quand deux personnes entrent dans le cercle (la roda) pour l'échange, on dit qu'elles «jouent» un «jeu». Dans le cercle protecteur de la musique et des témoins, le «jeu» commence par une double poignée de mains un geste d'échange et un toucher qui indiquent un accord tacite de jouer selon les règles («regarde, je ne cache pas de lame dans ma main»). Ce signe installe également un contexte d'engagement réciproque qui dit que je vais essayer de bien jouer pour que toi aussi tu puisses donner ton meilleur. Ce don d'une danse (ou d'un jeu) est une occasion de devenir et non de prouver que l'on est déjà quelqu'un. C'est un risque, mais les bénéfices qu'on en retire sont énormes. Browning voit la chose ainsi:

In a tight, «inside game» (jogo de denteo) when the players are interweaving spinning kicks, the agility and precision of one opens a precise space for the elegant partnering of the other. 9 
Cet engagement à jouer ensemble fait aussi partie du don-abandon de l'identité. C'est la volonté de changer constamment de position, de brouiller la distinction entre celui qui mène et celui qui est mené, celui qui attaque et celui qui évite. Comme dans le contact improvisation, pour atteindre à cette flexibilité dans le jeu, il est capital de décrire des mouvements circulaires. Ils ouvrent la voie fluide dans laquelle une attaque peut insensiblement devenir une défense et une esquive se changer en manœuvre offensive. C'est ainsi qu'un "compaso" peut dans sa course devenir une «negativa» dans cet univers de la roda qui passe constamment du combat à la danse.

Ce mélange de collaboration et de guerre fait partie de l'histoire de la Capoeira. Cela fait aussi partie, prétend Browning, de ses caractéristiques fondamentales:

But the strategic blending of fight and dance occurred in Brazil under specific pressures. And while this strategy appears to have been directed against forces outside the roda de capoeira, it became the fundamental strategy within the game. Dance - as seduction, illusion, deception - became dangerous, and kicks became elements of choreography. 10

Le texte que Browning a consacré à la Capoeira est judicieusement intitulé « Capoeira's Ironic Inversions» et elle perçoit toutes les roulades arrière, les sauts sur les mains, les roulades de tête et autres formes de mouvements inversés comme emblématiques de la capacité qu'a la Capoeira de voir le monde la tête en bas. Je les vois pour ma part comme faisant partie de la double vision de la Capoeira, un signe de sa volonté de survivre à son propre moment historique et de continuer à évoluer. C'est aussi une stratégie d'altérité, une façon d'être en dehors du système. Ce que veut montrer Browning dans la figure du capoeirista la tête à l'envers, c'est le fait qu'il ou elle accepte d'être désorienté(e). Pour moi, une des correspondances les plus intéressantes qu'il y a entre le contact improvisation et la Capoeira, c'est que les deux forment leurs praticiens à la désorientation - c'est-àdire les entraînent à se sentir à l'aise la tête en bas, en déséquilibre, pris de vertige ou lorsqu'ils se sentent tout simplement maladroits. Ces formes de danse entraînent leurs praticiens à surmonter la panique que l'on éprouve habituellement lorsqu'on se trouve dans des positions inhabituelles, en contrôlant les réactions immédiates du corps (tendre les muscles, retenir son souffle, ou fermer les yeux) pour leur substituer le désir d'éprouver la sensation que donne le fait d'être la tête en bas ou tout retourné. Cette volonté d'accepter d'être pris au dépourvu, sans défense, de courir des risques, de se mettre dans des situations inhabituelles, est liée à ce qui constitue la dimension de l'improvisation proprement dite dans la Capoeira et le contact improvisation.

L'improvisation est un phénomène très mal compris, en particulier dans le monde de la danse. Perçue comme le contraire de la chorégraphie, elle est souvent vue comme synonyme de liberté, de spontanéité, d'absence de technique ou de son oubli, de sauvagerie ou d'enfance, comme si lorsqu'on improvise on perdait tout simplement sa formation physique et esthétique pour devenir une case vide dans laquelle une imagination débridée peut «s'en donner à cour joie». Bien sûr, un improvisateur chevronné sait (qu'il s'agisse de danse, de musique ou de Capoeira) que l'improvisation nécessite un entraînement précis et rigoureux pour ouvrir l'imagination à de nouvelles possibilités narratives, libérer le corps de ses réactions habituelles et éveiller la curiosité de se lancer dans l'inconnu. C'est aussi dans l'improvisation que l'entraînement à la désorientation s'avère capital. Nous percevons souvent le préfixe de «dés/orientation» comme négatif, désignant une absence d'orientation. Mais on pourrait réinterpréter le mot comme désignant plutôt une autre sorte d'orientation, comme celle que l'on éprouve, par exemple, en voyant le monde la tête en bas. Nous oublions parfois, dans notre mode de fonctionnement linéaire, visuel, cérébral, qu'il existe de nombreuses autres stratégies d'organisation qui ne ressemblent pas nécessairement aux formes auxquelles nous sommes habitués. Dans le contact improvisation et la Capoeira, l'improvisation libère l'individu d'une certaine façon d'ordonner le monde afin de lui offrir 
la possibilité d'un «autre» genre d'ordonnancement, souvent nettement moins visible. C'est ainsi qu'une structure de mouvement aussi simple que marcher à reculons peut forcer la personne à adopter d'autres réflexes et d'autres positions (regarder entre ses jambes, par exemple) pour s'orienter. Même s'il existe de nombreux exercices différents pour développer ses capacités d'improvisateur, le véritable test se passe quand on entre dans le cercle pour rejoindre son partenaire. C'est en effet le moment où tout le monde - du débutant au praticien le plus accompli - doit faire un acte de foi et se lancer dans l'inconnu.

Les toutes premières secondes sont les plus difficiles, car elles ouvrent l'espace de la rencontre, de la compréhension réciproque, de la perception de ses propres rythmes, de son poids, de son hésitation ou de sa force. C'est un moment effrayant mais heureusement il ne se produit pas dans le vide. Dans la Capoeira, la roda crée un cercle protecteur qui non seulement met les joueurs à l'abri de toute interférence extérieure mais maintient aussi l'attention de ceux qui le constituent. Dans le contact improvisation, de la même façon, la manifestation constitue un cercle fermé de spectateurs dont l'attention concentrée sur les événements qui se déroulent au centre aide à conserver une atmosphère presque sacrée. Les deux formes de danse s'avèrent très soucieuses de garder les cercles suffisamment réguliers dans leur disposition pour qu'il ne s'y produise pas de trou noir ou de brèche dans le cercle des énergies. C'est une question de sécurité, bien sûr, mais l'énergie cohésive du cercle produit aussi une mystérieuse attention qui vient chercher la magie de ces interactions et pousse les participants à donner leur pleine mesure. Mystérieuse certes, mais fort agréable, comme vous le dira quiconque a jamais assisté à une bonne jam ou à une bonne roda.

Ce cercle d'attention commence à se former comme un espace sauvage - un espace d'improvisation, un espace qui célèbre l'acte de devenir (ensemble) quelque chose d'imprévu. C'est un anneau de continuité qui offre la possibilité d'une transformation et d'un échange entre les gens. Je propose d'y voir un espace intersubjectif. Non pas parce que, de façon magique, il transformerait la différence en quelque chose qui ne fait pas de différence (soit en effaçant les différences soit au contraire en les multipliant de façon exponentielle). Non, c'est un espace intersubjectif parce que lorsque j'y pénètre avec une autre personne (le duo du contact improvisation, le jeu de la Capoeira), je rencontre l'«autre» en moi. Mais je ne m'arrête pas là, figé par cette image de l'altérité qui me fait face. Je traverse le miroir et je passe des images bidimensionnelles au mouvement tridimensionnel. Me joindre au mouvement d'un autre me libère d'une position de subjectivité qui exige d'avoir un objet pour prédicat. Cela me permet d'aller au-delà de la connaissance que j'ai de moi-même pour faire l'expérience de mon identité prise au beau milieu des énergies d'un autre. Cette volonté de fusionner avec les énergies, les mouvements, les rythmes, les idées d'un autre est une des leçons les plus riches que l'on puisse tirer aussi bien du contact improvisation que de la Capoeira, et c'est quelque chose que je travaille à traduire dans les ateliers intitulés «Engaging Bodies: the Politics and Poetics of Corporeality» que je donne pour les universitaires. Savoir négocier l'intimité du combat ou du contact physique m'a fait prendre conscience de la valeur du savoir que renferme mon corps et m'a aidée à voir que nous devons trouver une forme de communication plus détendue, faire appel à d'autres formes d'intelligence corporelle pour ouvrir nos corps, nos imaginations et réussir à traiter toutes les questions que pose la différence culturelle.

En ce sens, le contact improvisation et la Capoeira sont des formes hybrides qui peuvent nous fournir d'importants modèles pour mieux vivre le $21^{\mathrm{e}}$ siècle, car elles sont hybrides de deux façons. Culturellement parce que, avec les années, elles se sont littéralement incorporé une information sur le mouvement qui leur vient de la danse moderne et postmoderne, du hip-hop, des arts martiaux asiatiques et d'autres influences encore, trop nombreuses pour être énumérées ici. Cette capacité d'adaptation a non seulement gardé ces formes vivantes à travers diverses situations, mais elle a aussi contredit les notions traditionnelles 
d'authenticité culturelle, de continuité historique, de nation et de communauté. Mais ce qui est le plus important pour mon propos ici, c'est que ces formes sont aussi hybrides intérieurement, en ce sens qu'elles mettent l'accent sur la rencontre entre deux personnes, deux sources d'énergie, deux préférences de mouvement, chaque fois que l'on active leurs cercles respectifs. Les deux formes de danse orchestrent une confrontation avec une "autre" personne mais aussi avec l'«autre» en moi. Mais cette rencontre prend place dans un ethos basé sur la possibilité du mouvement et du changement. Dans cet espace, les divers corps, les diverses énergies, les diverses positions (du sujet) vont et viennent (comme le ginga ou le point de contact en mouvement) et changent si vite qu'on ne parvient à discerner que le mouvement qui circule entre eux et les unit. Les pôles du moi et de l'autre (que l'on peut schématiser par des lignes parallèles) perdent leur orientation et leur sens souvent oppositionnels lorsque la vibration entre eux devient la force électrique qui anime un autre genre d'échange (en forme, cette fois, de X ou de croix de Saint-André).

\section{N O TES}

1. S. Smith, Subjectivity, Identity and the Body, Bloomington, Indiana University Press, 1993.

2. J. Butler, «Variations on Sex and Gender: Beauvoir, Wittig and Foucault", dans Feminism as Critique: On the politics of Gender, S. Benhabib et D. Cornell (dir.), Minneapolis, University of Minnesota Press, 1987, p. 133. [En définissant les femmes comme "Autre», les hommes parviennent, par le raccourci de la définition, à disposer de leurs corps en se faisant, eux, du même coup, autre chose que leurs corps (...) Il n'est dès lors guère difficile, à partir de cette idée que le corps est Autre, de sauter à la conclusion que les autres sont leurs corps tandis que le «je» masculin est l'âme incorporelle. Le corps considéré comme Autre - le corps réprimé ou nié puis par la suite projeté - resurgit pour ce «je » sous la forme de cette idée que les autres sont essentiellement corps. C'est ainsi que les femmes deviennent l'Autre; elles en viennent à incarner la corporéité même.]

3. À la fin des années soixante-dix et au début des années quatre-vingt, les vétérans qui pratiquaient cette forme depuis dix ans avaient passablement maîtrisé un style de danse virtuose offrant des caractéristiques de fluidité et de souplesse ainsi qu'une dimension acrobatique qui paraissait souvent délicieusement dangereuse, par exemple lorsque l'un des danseurs effectuait une sorte de spirale audessus de l'autre pour venir retomber en vrille sur ses épaules. Passés maîtres dans l'art de la chute, de la roulade et du saut à haute vitesse, ces danseurs se concentraient sur les dynamiques physiques les plus abstraites des mouvements plutôt que sur les implications sociales de la danse. À la fin des années quatre-vingt et dans les années quatre-vingtdix, de nombreux pratiquants du contact improvisation ont donné une plus grande ampleur à cette forme de danse en faisant appel à ses implications émotionnelles, théâtrales et politiques. L'initiation au contact improvisation de jeunes enfants, de personnes âgées et de personnes souffrant d'un handicap a représenté un facteur clé de ce nouveau développement. Voir à ce sujet le livre de C. Novack: Sharing the Dance, Madison, University of Wisconsin Press, 1990, et la revue Contact Quarterly, en particulier le numéro de l'hiver 1992.

4. B. Browning, "Headspin: Capoeira's Ironic Inversions", dans Samba: Resistance in Motion, Bloomington, Indiana University Press, 1995, p. 108 [le non au cour du oui, le grand dans le petit, la terre dans le ciel, le combat dans la danse.]

5. D. Williams, «(In) the In-Between", dans Writings on Dance, hiver 1996, p. 26 [un entre-deux ou une entremise, (c')est un autre espace dans lequel le "Je" se trouve à la fois impliqué et (re)conçu; c'est l'articulation d'une rencontre dans la différence.]

6. Ibid, p. 26 [Pour chacun des partenaires, le contact constitue la possible coexistence de la forme et de la spontanéité, des règles du jeu et de la danse, de la cause et de l'effet, du centre et de la marge, de la proximité et de la distance. Il représente le "jeu», le "don", dans l'inflexible fixité des identités physiques; il en est le souple-ément (sic), la différance: les frontières instables où naît une éthique de l'altérité.] 7. Ibid, p. 25 (c'est moi qui souligne). [Le facteur capital ici, ce n'est pas le nombre de façons différentes dont deux identités distinctes peuvent se combiner, mais la reconnaissance que ce «troisième élément" n'est pas une unité mais un axe, pas une entité mais une façon d'être, moins une relation que l'acte de se relier.]

8. E. Grosz, Volatile Bodies, Bloomington, Indiana University Press, 1994, p. 209 [l'altérité n'est jamais que la possibilité même et le processus de l'incarnation.]

9. Browning, op. cit., p. 90 [Dans un jeu serré, pour connaisseurs, quand les joueurs enlacés s'échangent des semblants de coups entrecroisés, l'agilité et la précision de l'un ouvrent un espace précis pour la participation de l'autre au partenariat.]

10. Ibid, p. 91 [Mais ce mélange stratégique de combat et de danse s'est constitué, au Brésil, sous des pressions spécifiques. Et même si cette stratégie semble avoir été dirigée contre des forces situées à l'extérieur de la roda de capoeira, elle est devenue la stratégie fondamentale utilisée à l'intérieur du jeu. La danse - en tant que séduction, illusion, déception - est devenue dangereuse et les coups sont devenus des éléments de chorégraphie.] 